\title{
Structure of the Global Attractors in a Model for Ectoparasite-Borne Diseases
}

\author{
Attila Dénes* and Gergely Röst* \\ * Bolyai Institute, University of Szeged, \\ Aradi vértanúk tere 1, Szeged, Hungary \\ Emails: denesa@math.u-szeged.hu, rost@math.u-szeged.hu
}

Received: 15 July 2012, accepted: 25 September 2012, published: 19 October 2012

\begin{abstract}
We delineate a mathematical model for the dynamics of the spread of ectoparasites and the diseases transmitted by them. We present how the dynamics of the system depends on the three reproduction numbers belonging to three of the four possible equilibria and give a complete characterization of the structure of the global attractor in each possible case depending on the reproduction numbers.
\end{abstract}

Keywords-ectoparasites; global dynamics; global attractors

\section{INTRODUCTION}

Ectoparasites (e.g. lice, fleas, mites) cause a serious problem in several parts in the world [2], [4]. Ectoparasite infestations are often connected to the lack of hygiene and poor economical conditions, however, their presence is increasing in developed countries as well.

The three louse species which transmit diseases are the head louse, the body louse and the pubic louse. These species are responsible for the spread of trench fever, epidemic typhus and relapsing fever. The flea species which most commonly affect humans are the cat, the rat and the human flea. Fleas transmit plague, murine typhus, fleaborne spotted rickettsiosis. The transmission of these diseases is different from that of other vectorborne diseases, as it is carried out through the human contact network, which means that the spread of the vectors themselves is similar to that of a disease.

In this paper we delineate a model for the dynamics of ectoparasite-borne diseases and we describe the structure of the global attractors in the different situations depending on the reproduction numbers. We assume the presence of one disease and one ectoparasite species which is a vector transmitting this particular disease.

The human population is divided into three compartments: susceptibles (i.e. those who can be infested by both infectious and non-infectous vectors, denoted by $S(t)$ ), those who are infected by non-infectious parasites (denoted by $T(t)$ ) and those who are infested by infectious vectors (denoted by $Q(t)$ ). We assume that someone infested by non-infectious vectors can transmit the parasites to susceptibles, while an individual infested by infectious vectors transmits both the parasites and the disease to susceptibles. An individual infested by infectious vectors transmits the infection to individuals infested by non-infectious vectors, i.e. a member of compartment $T$ can move to compartment $Q$ upon adequate contact with someone from compartment $Q$. We assume that a person is infected by the disease if and only he is infested by infectious parasites. We suppose that individuals infested by infected parasites transmit the disease at the same rate to susceptibles and to those who are infested by non-infected parasites. We denote this transmission rate by $\beta_{Q}$, and $\beta_{T}$ denotes the transmission rate for non-infectious vectors (to susceptibles). The rate of disinfestation is denoted by $\mu$ for the infected compartment and by $\theta$ for the non-infected compartment. We denote by $b$ the natural birth and death rates, and we assume the disease is not fatal, thus the population size is constant. In the model equations we use mass action incidence.

We have the following system of differential equations 


\section{A. Dénes et al., Structure of the Global Attractors in a Model for Ectoparasite-Borne Diseases}

(with all the parameters assumed to be positive):

$$
\begin{aligned}
S^{\prime}(t) & =-\beta_{T} S(t) T(t)-\beta_{Q} S(t) Q(t) \\
& +\theta T(t)+\mu Q(t)+b-b S(t), \\
T^{\prime}(t) & =\beta_{T} S(t) T(t) \\
& -\beta_{Q} Q(t) T(t)-\theta T(t)-b T(t), \\
Q^{\prime}(t) & =\beta_{Q} S(t) Q(t) \\
& +\beta_{Q} Q(t) T(t)-\mu Q(t)-b Q(t) .
\end{aligned}
$$

It can easily be seen that any solution with nonnegative initial values remains non-negative for all forward time. We can suppose that

$$
N(t)=S(t)+T(t)+Q(t)=1
$$

holds for the total population. The phase space of our system is $X:=\left\{(S, T, Q) \in \mathbb{R}_{+}^{3}: S+T+Q=1\right\}$.

\section{EQUILIBRIA, REPRODUCTION NUMBERS}

By solving the algebraic equations

$$
\begin{aligned}
& 0=-\beta_{T} S^{*} T^{*}-\beta_{Q} S^{*} Q^{*}+\theta T^{*}+\mu Q^{*}+b-b S^{*}, \\
& 0=\beta_{T} S^{*} T^{*}-\beta_{Q} Q^{*} T^{*}-\theta T^{*}-b T^{*}, \\
& 0=\beta_{Q} S^{*} Q^{*}+\beta_{Q} Q^{*} T^{*}-\mu Q^{*}-b Q^{*},
\end{aligned}
$$

we can determine the four equilibria of system (1):

$$
\begin{aligned}
E_{S} & =(1,0,0), \\
E_{T} & =\left(\frac{b+\theta}{\beta_{T}}, 1-\frac{b+\theta}{\beta_{T}}, 0\right), \\
E_{Q} & =\left(\frac{b+\mu}{\beta_{Q}}, 0,1-\frac{b+\mu}{\beta_{Q}}\right), \\
E_{Q T} & =\left(\frac{\theta-\mu+\beta_{Q}}{\beta_{T}}, \frac{b+\mu}{\beta_{Q}}-\frac{\theta-\mu+\beta_{Q}}{\beta_{T}}, 1-\frac{b+\mu}{\beta_{Q}}\right) .
\end{aligned}
$$

Reproduction numbers have a clear biological interpretation. We can obtain them by multiplying the number of new infections and the average length of the infectious period of an infectious agent newly introduced into a population currently being in one of the equilibria.

By introducing an infested, non-infectious individual into a population in the equilibrium $E_{S}$, we obtain the reproduction number

$$
R_{1}=\frac{\beta_{T}}{b+\theta},
$$

by introducing an infested and infectious individual into the same equilibrium we obtain the reproduction number

$$
R_{2}=\frac{\beta_{Q}}{b+\mu} .
$$

Calculating the expected number of secondary infections caused by the introduction of an infectious infested individual into a population in the equilibrium $E_{T}$ gives the same reproduction number $R_{2}$.
If we introduce a non-infectious infested individual into a population in the equilibrium $E_{Q}$, we obtain the reproduction number

$$
R_{3}=\frac{\beta_{T}(b+\mu)}{\beta_{Q}\left(\beta_{Q}-\mu+\theta\right)} .
$$

The following lemma is taken from [3].

Lemma 2.1: The equilibrium $E_{S}$ always exists. The equilibrium $E_{T}$ exists if and only $R_{1}>1$. The equilibrium $E_{Q}$ exists if and only if $R_{2}>1$. The equilibrium $E_{Q T}$ exists if and only if $R_{2}>1$ and $R_{3}>1$.

\section{Structure of the Global Attractor}

Here we recall the main result of [3].

Theorem 3.1: Let $X_{Q}:=\left\{(S, T, 0) \in \mathbb{R}_{+}^{3}: S+T=1\right\}$ and $X_{T}:=\left\{(S, 0, Q) \in \mathbb{R}_{+}^{3}: S+Q=1\right\}$ denote the extinction sets for $Q$ and $T$, respectively. The four equilibria have the following global stability properties depending on the reproduction numbers:

(i) Equilibrium $E_{S}$ is globally asymptotically stable if $R_{1} \leq$ 1 and $R_{2} \leq 1$.

(ii) Equilibrium $E_{T}$ is globally asymptotically stable on $X \backslash$ $X_{T}$ if $R_{1}>1$ and $R_{2} \leq 1$. On $X_{T}, E_{S}$ is globally asymptotically stable.

(iii) If $R_{2}>1, R_{3} \leq 1$ and $R_{1} \leq 1$, then $E_{Q}$ is globally asymptotically stable on $X \backslash X_{Q}$ and $E_{S}$ is globally asymptotically stable on $X_{Q}$.

(iv) If $R_{2}>1, R_{3} \leq 1$ and $R_{1}>1$, then $E_{Q}$ is globally asymptotically stable on $X \backslash X_{Q}$ and $E_{T}$ is globally asymptotically stable on $X_{Q}$.

(v) If $R_{2}>1, R_{3}>1$, then $E_{Q T}$ is globally asymptotically stable on $X \backslash\left(X_{Q} \cup X_{T}\right), E_{T}$ is globally asymptotically stable on $X_{Q}$ and $E_{Q}$ is globally asymptotically stable on $X_{T}$.

An equilibrium $E$ is said to be globally asymptotically stable on a set $Y$ if it is stable and for all $y \in Y$ the solution starting from $y$ converges to $E$ as $t \rightarrow \infty$. Following the notation of [1. 1.1.7], by $M t$ we denote the set consisting of the states at time $t$ of the solutions started from all of the points $x \in M$.

Definition 3.2: Let $\mathcal{A} \in X$ be a compact invariant set. If $\mathcal{A}$ attracts each bounded subset of $X$, i.e. for any bounded subset $M \subset X$ and any neighbourhood $U$ of $\mathcal{A}$ there exists a $T<\infty$ such that $M t \subset U$ for all $t>T$, then $\mathcal{A}$ is called the global attractor.

Definition 3.3: The $\omega$-limit set of a point $x \in X$, denoted by $\omega(x)$ consists of those elements $y$ of $X$ for which there exists a real sequence $\left\{t_{n}\right\}$ such that $t_{n} \nearrow \infty$ and $x t_{n} \rightarrow y$ as $n \rightarrow \infty$. The $\alpha$-limit set is defined similarly with $t_{n} \searrow-\infty$.

In the following theorem we describe the structure of the global attractor for system (1) in the five cases listed in Theorem 3.1 .

Theorem 3.4: The global attractor $\mathcal{A}$ for system (1) has the following structure:

(i) $\mathcal{A}=\left\{E_{S}\right\}$. 


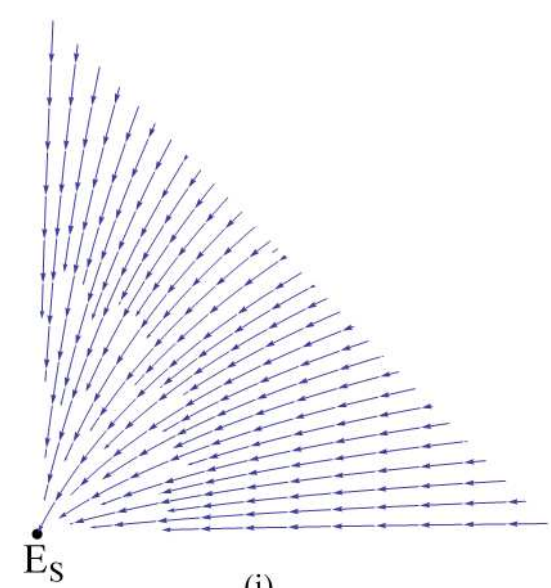

(i)

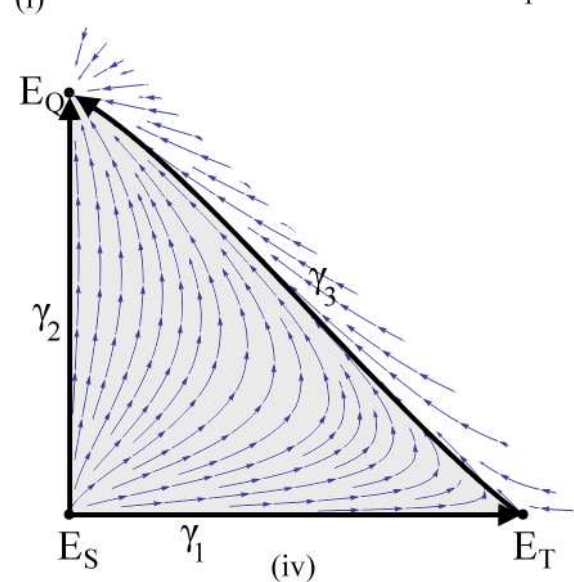

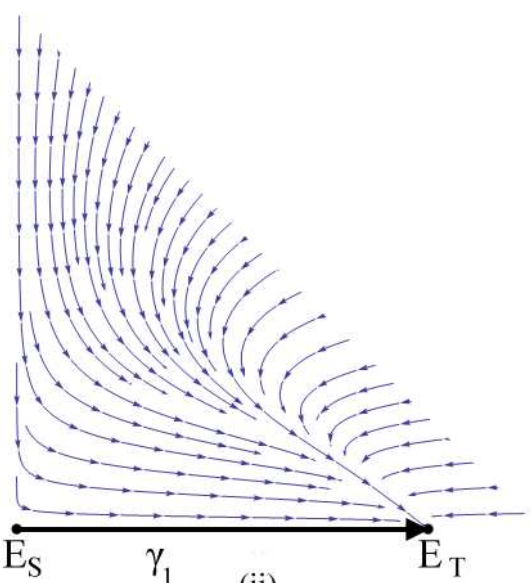

(ii)

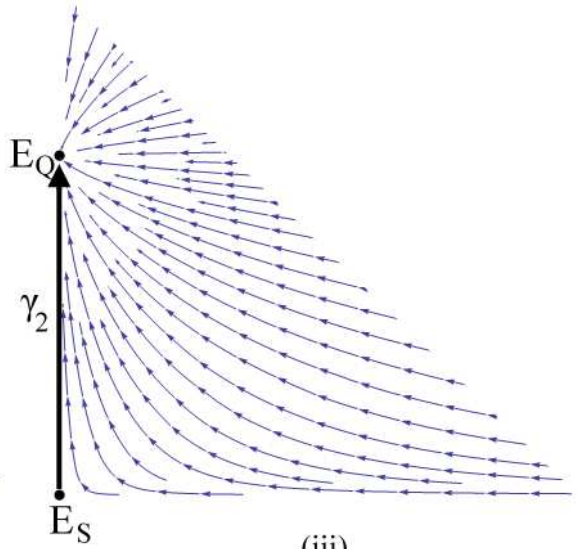

(iii)

Fig. 1. Representation of the flow and the attractors on the $T Q$-plane.

(ii) $\mathcal{A}=\left\{E_{S}, E_{T}\right\} \cup \gamma_{1}$, where $\gamma_{1}$ is a connecting orbit from $E_{S}$ to $E_{T}$, which is actually the segment between $E_{S}$ and $E_{T}$ in the extinction space $X_{Q}$.

(iii) $\mathcal{A}=\left\{E_{S}, E_{Q}\right\} \cup \gamma_{2}$, where $\gamma_{2}$ is a connecting orbit from $E_{S}$ to $E_{Q}$, which is actually the segment between $E_{S}$ and $E_{Q}$ in the extinction space $X_{T}$.

(iv) $\mathcal{A}=\left\{E_{S}, E_{T}, E_{Q}\right\} \cup \gamma_{1} \cup \gamma_{2} \cup \gamma_{3} \cup \mathcal{A}_{1}$, where $\gamma_{3}$ is a connecting orbit from $E_{T}$ to $E_{Q}$, and $\mathcal{A}_{1}$ is the domain surrounded by $E_{S}, E_{T}, E_{Q}, \gamma_{1}, \gamma_{2}$ and $\gamma_{3}$ in the $T Q$ plane consisting of connecting orbits from $E_{S}$ to $E_{Q}$.

(v) $\mathcal{A}=\left\{E_{S}, E_{T}, E_{Q}, E_{Q T}\right\} \cup \gamma_{1} \cup \gamma_{2} \cup \gamma_{4} \cup \gamma_{5} \cup \mathcal{A}_{2}$, where $\gamma_{4}$ is a connecting orbit from $E_{T}$ to $E_{Q T}, \gamma_{5}$ is a connecting orbit from $E_{Q}$ to $E_{Q T}$, and $\mathcal{A}_{2}$ is the domain surrounded by $E_{S}, E_{T}, E_{Q}, E_{Q T}, \gamma_{1}, \gamma_{2}, \gamma_{4}$ and $\gamma_{5}$ in the $T Q$-plane consisting of connecting orbits from $E_{S}$ to $E_{Q T}$.

Proof:

(i) As proved in Theorem $3.1, E_{S}$ is globally asymptotically stable in case (i), which means that the global attractor is the singleton $E_{S}$ in this case.

For the proof of the remaining cases we reduce the system to two dimensions by substituting $S$ with $1-T-Q$.
We get the system

$$
\begin{aligned}
T^{\prime}(t) & =\beta_{T}(1-T(t)-Q(t)) T(t) \\
& -\beta_{Q} Q(t) T(t)-\theta T(t)-b T(t), \\
Q^{\prime}(t) & =\beta_{Q}(1-T(t)-Q(t)) Q(t) \\
& +\beta_{Q} Q(t) T(t)-\mu Q(t)-b Q(t)
\end{aligned}
$$

and the four equilibria

$$
\begin{aligned}
E_{S} & =(0,0), \\
E_{T} & =\left(1-\frac{b+\theta}{\beta_{T}}, 0\right), \\
E_{Q} & =\left(0,1-\frac{b+\mu}{\beta_{Q}}\right), \\
E_{Q T} & =\left(\frac{b+\mu}{\beta_{Q}}-\frac{\theta-\mu+\beta_{Q}}{\beta_{T}}, 1-\frac{b+\mu}{\beta_{Q}}\right) .
\end{aligned}
$$

By standard linearization, we calculate the eigenvalues and eigenvectors of the Jacobian of the linearized system in the four equilibria. The details of the calculations are straightforward thus omitted, here we only discuss the results and implications. The eigenvalues of the Jacobian 


\section{A. Dénes et al., Structure of the Global Attractors in a Model for Ectoparasite-Borne Diseases}

of the linearized equation around the equilibrium $E_{S}$ are $\lambda_{1}=-b-\theta+\beta_{T}=(b+\theta)\left(R_{1}-1\right)$ with corresponding eigenvector $(1,0)$ and $\lambda_{2}=-b-\mu+\beta_{Q}=(b+\mu)\left(R_{2}-\right.$ $1)$ with corresponding eigenvector $(0,1)$. Linearizing at the equilibrium $E_{T}$, one finds the eigenvalues $\lambda_{1}=b+$ $\theta-\beta_{T}=(b+\theta)\left(1-R_{1}\right)$ with the eigenvector $(1,0)$ and $\lambda_{2}=-b-\mu+\beta_{Q}=(b+\mu)\left(R_{2}-1\right)$ with the eigenvector

$$
\left(\frac{\left(\beta_{Q}+\beta_{T}\right)\left(b+\theta-\beta_{T}\right)}{\beta_{T}\left(\beta_{Q}+\beta_{T}-2 b-\theta-\mu\right)}, 1\right) .
$$

Linearization around the steady state $E_{Q}$ gives the following eigenvalues of the Jacobian: $\lambda_{1}=b+\mu-\beta_{Q}=$ $(b+\mu)\left(1-R_{2}\right)$ with the eigenvector $(0,1)$ and $\lambda_{2}=$ $-\theta+\mu-\beta_{Q}+(b+\mu) \beta_{T} / \beta_{Q}=\left(R_{3}-1\right) \beta_{T} /\left(R_{2} R_{3}\right)$ with the eigenvector $(1,0)$. Finally, if we linearize the system around the equilibrium $E_{Q T}$, we obtain the eigenvalues $\lambda_{1}=b+\mu-\beta_{Q}=(b+\mu)\left(1-R_{2}\right)$ with corresponding eigenvector

$\left(\frac{\left(\beta_{Q}+\beta_{T}\right)\left(\beta_{Q}\left(\beta_{Q}+\theta\right)-b \beta_{T}-\mu\left(\beta_{Q}+\beta_{T}\right)\right)}{\beta_{T}\left(b\left(\beta_{Q}+\beta_{T}\right)-\beta_{Q}\left(2 \beta_{Q}+\theta\right)+\left(2 \beta_{Q}+\beta_{T}\right) \mu\right)}, 1\right)$ and $\lambda_{2}=\theta-\mu+\beta_{Q}-(b+\mu) \beta_{T} / \beta_{Q}=(1-$ $\left.R_{3}\right) \beta_{T} /\left(R_{2} R_{3}\right)$ with corresponding eigenvector $(1,0)$.

(ii) If $R_{1}>1$ and $R_{2}<1$ then $E_{S}$ has the stable eigenvector $(0,1)$ and the unstable eigenvector $(1,0)$. This means that $E_{S}$ has a one-dimensional stable manifold which coincides with the invariant extinction space $X_{T}$ and a one-dimensional unstable manifold which coincides with the segment $\left(E_{S}, E_{T}\right)$ of the extinction space $X_{Q}$, while both of the eigenvectors at $E_{T}$ are stable. $\gamma_{1}$ is the connecting orbit from $E_{S}$ to $E_{T}$ lying in $X_{Q}$. If $R_{2}=1$ then the second eigenvalue at $E_{S}$ is equal to zero. In this case, the equation for $Q^{\prime}(t)$ takes the form

$$
Q^{\prime}(t)=-\beta_{Q} Q^{2}(t)<0
$$

on $X_{T}$, which means that all solutions started from $X_{T}$ tend to $E_{S}$. Thus $E_{S}$ has the same one-dimensional stable and unstable sets as in the case $R_{2} \neq 1$. From Theorem 3.1 we know that all solutions started from $X \backslash X_{T}$ tend to $E_{T}$, thus $E_{T}$ has a two-dimensional stable set.

(iii) If $R_{1}<1, R_{2}>1$ and $R_{3}<1$ then $E_{S}$ has the stable eigenvector $(1,0)$, and the unstable eigenvector $(0,1)$, while $(0,1)$ and $(1,0)$ are both stable eigenvectors for $E_{Q}$. If $R_{1}=1$ then the equation for $T^{\prime}(t)$ takes the form

$$
T^{\prime}(t)=-\beta_{T} T^{2}(t)<0,
$$

on the invariant extinction space $X_{Q}$. This means that all solutions on the center manifold belonging to the zero eigenvalue (which coincides with $X_{Q}$ ) tend to $E_{S}$. If $R_{3}=1$ then the Jacobian of the linearized system at $E_{Q}$ has a zero eigenvalue with eigenvector $(1,0)$. The line

$$
Q=1-\frac{b+\mu}{\beta_{Q}}
$$

is invariant: if we substitute $1-(b+\mu) / \beta_{Q}$ into the equation for $Q^{\prime}(t)$ we get $Q^{\prime}(t)=0$. This means that for $R_{3}=1$ the center manifold belonging to the zero eigenvalue coincides with this line. For $R_{3}=1$, the equation for $T^{\prime}(t)$ has the form

$$
T^{\prime}(t)=-\beta_{T} T^{2}(t)<0
$$

on this line, which means that all solutions started from this line tend to the equilibrium $E_{Q} \cdot \gamma_{2}$ is the connecting orbit from $E_{S}$ to $E_{Q}$ lying in $X_{T}$. This shows the statement of (iii).

(iv) In this case, the first eigenvector belonging to $E_{S}$ loses its stability, while the same vector becomes a stable eigenvector for $E_{T}$. Thus, $E_{S}$ has two unstable eigenvectors and $E_{T}$ has the stable eigenvector $(1,0)$ and an unstable eigenvector. From Theorem 3.1 we know that any solution started from the one-dimensional unstable manifold of $E_{T}$ tends to $E_{Q}$, from which the existence of a heteroclinic orbit $\gamma_{3}$ from $E_{T}$ to $E_{Q}$ follows. The situation for $E_{Q}$ is the same as in case (iii). We have to show that $\mathcal{A}_{1}$ consists of heteroclinic orbits from $E_{S}$ to $E_{Q}$. Let us take an arbitrary point $p \in \mathcal{A}_{1}$. From Theorem 3.1 we know that $\omega(p)=\left\{E_{Q}\right\}$. The negative limit set $\alpha(p)$ exists and is non-empty as the backward orbit is bounded by $\gamma_{1} \cup \gamma_{2} \cup \gamma_{3}$. From the Poincaré-Bendixson Theorem we know that $\alpha(p)$ can only be an equilibrium point (as there are no periodic orbits). We can exclude $E_{Q}$ as it has a two-dimensional stable manifold. The unstable manifold of $E_{T}$ coincides with $X_{Q}$, which is invariant and $\mathcal{A}_{1} \cap X_{Q}=\emptyset$, thus $\alpha(p)=\left\{E_{S}\right\}$.

(v) In this case, again, $E_{S}$ has two unstable eigenvectors and thus a two-dimensional unstable manifold. Similarly to the previous case, $E_{T}$ has a stable and an unstable eigenvector, thus having a one-dimensional stable manifold and a one-dimensional unstable manifold. The eigenvector $(1,0)$ for $E_{Q}$ is unstable, which means that $E_{Q}$ has a one-dimensional stable manifold and a one-dimensional unstable manifold. $E_{Q T}$ has two stable eigenvectors and thus a two-dimensional stable manifold. From Theorem 3.1 we know that all solutions started from $X \backslash\left(X_{T} \cup X_{Q}\right)$ tend to $E_{Q T}$, thus there exists a connecting orbit $\gamma_{4}$ from $E_{T}$ to $E_{Q T}$ and a connecting orbit $\gamma_{5}$ from $E_{Q}$ to $E_{Q T}$. Similarly to case (iv) we can show that the domain $\mathcal{A}_{2}$ consists of connecting orbits from $E_{S}$ to $E_{Q T}$.

If neither is available on your system, please use the font closest in appearance to Times. Avoid using bit-mapped fonts if possible. True-Type 1 or Open Type fonts are preferred. Please embed symbol fonts, as well, for math, etc.

\section{CONCLUSION}

We described the global attractor in all possible cases. Depending on the three reproduction numbers, the global attractor might have the following structure: 


\section{A. Dénes et al., Structure of the Global Attractors in a Model for Ectoparasite-Borne Diseases}

- a singleton

- a one-dimensional set consisting of two equilibria and a connecting orbit

- a two-dimensional set consisting of three or four equlibria and connecting orbits between them.

The biological interpretation of our results is the following. The reproduction numbers $R_{i}(i=1,2,3)$ completely determine whether the infectious or the non-infectious parasites can invade a human population. This is mathematically expressed in the structure of the global attractors that we described. If $R_{1} \leq 1$ and $R_{2} \leq 1$, then the population is safe from any parasites. The implication for the control of the infection and infestation is that to eradicate the disease only, we have to decrease $R_{2}$ to be less than 1 , which is possible by reducing $\beta_{Q}$ or increasing $\mu$. To eliminate all the parasites, besides decreasing $R_{2}$ we also have to decrease $R_{1}$ (possible by reducing $\beta_{T}$ or increasing $\theta$ ). Decreasing only $R_{1}$ is not enough for the elimination of the parasites. The reproduction number $R_{3}$ is a threshold parameter which shows whether all the parasites become infectious or both infectious and noninfectious parasites can be present in the population. The transmission rates $\beta_{Q}$ and $\beta_{T}$ can be effectively reduced by vigorous monitoring and isolation of infested individuals, while $\mu$ and $\theta$ can be increased by disinfestation treatment of individuals.

\section{ACKNOWLEDGMENT}

Research supported by European Research Council Starting Grant Nr. 259559, OTKA K75517 and Bolyai Scholarship of Hungarian Academy of Sciences.

\section{REFERENCES}

[1] N. P. Bhatia, G. P. Szegö, Dynamical Systems: Stability Theory and Applications, Springer-Verlag, 1967.

[2] P. Brouqui, D. Raoult, "Arthropod-Borne Diseases in Homeless", Ann. N.Y. Acad. Sci., vol. 1078, pp. 223-235, 2006. http://dx.doi.org/10.1196/annals.1374.041

[3] A. Dénes, G. Röst, "Global dynamics for the spread of ectoparasite-borne diseases", submitted.

[4] L. Houhamdi, P. Parola, D. Raoult, "Les poux et les maladies transmises à l'homme", Med. Trop., vol. 65, pp. 13-23, 2005.

[5] H. L. Smith, H. R. Thieme, Dynamical Systems and Population Persistence, Graduate Studies in Mathematics, vol. 118, AMS, Providence, 2011. 